\title{
Erratum: Leidenfrost effect: Accurate drop shape modeling and refined scaling laws [Phys. Rev. E 90, 053011 (2014)]
}

\author{
B. Sobac, A. Rednikov, S. Dorbolo, and P. Colinet
}

(Received 15 February 2021; published 16 March 2021; corrected 30 March 2021)

DOI: 10.1103/PhysRevE.103.039901

Following the remarks by Chakraborty et al. [17], we discovered a typing mistake in our original numerical code treating the problem formulated in Sec. II of the original paper. Namely, in our code, in the numerator of the curvature expression Eq. (3), we incorrectly typed $1+r^{-1}\left(\partial_{r} h\right)^{2}$ instead of the correct $r^{-1}\left[1+\left(\partial_{r} h\right)^{2}\right]^{1}$. In the present erratum, we remake the figures of our paper containing the numerical results to be thereby corrected and point out the conclusions affected as a consequence, the original paper remaining unchanged in all other regards. The corrected numerical results are represented in Figs. 2-5 and 7, the figure numbering adhering to the original one of our paper.

One can see that the results are quite modestly affected and no major conclusions need to be reconsidered for midsized and large droplets $\left(R_{\max } \gtrsim 0.5\right)$. Perhaps, it is only worthwhile to note that we now obtain $R_{c}$ slightly larger than $R_{\text {neck }}$, whereas it was slightly smaller in the original paper, cf. Fig. 4 here and there. Besides, the increase in $h_{\text {center }}$ for larger droplets, precursor of chimney formation, is now noticeably less pronounced than it was in the original paper, cf. Figs. 3(b) and 7(d) here and there. The latter change is seen to improve the agreement with experiment in Fig. 3(b) for larger $R_{\max }$.

In contrast, the results are drastically affected for smaller droplets as already pointed out by Chakraborty et al. [17]. This concerns, first of all, the neck disappearance for $R_{\max }$ below a certain small value (dependent on $\mathcal{E}$ and at which $R_{\text {neck }} \rightarrow 0$ ), which we missed in our paper due to the numerical mistake. Thus, a small Leidenfrost droplet attains a practically spherical shape at not so small $R_{\max }$ as we originally thought. Another important modification is an eventual increase in $h_{\text {center }}$ as the droplet size is decreased below a certain $R_{\max }$ (whose value depends on $\mathcal{E}$ ), cf. Figs. 5(c) and 5(d), which can be seen as a precursor of the Leidenfrost droplet take-off studied. In this domain of smaller droplets, the correction of the numerical results renders the agreement with experiment less good than it happened to be before for the wrong reasons, cf. Fig. 3, although note that the unfilled circles in Fig. 3(b) seem to remotely reproduce the theoretical tendency given by the solid and dashed curves. No scaling is now appropriate and plotted in Figs. 5(c) and 5(d) for $h_{\text {neck. }}$. Rather, the solid curve and dashed line newly incorporated in the range of smaller droplets represent an asymptotic result for a spherical Leidenfrost particle in the limit $h_{\text {center }} \ll R_{\max }$ (see the Appendix for more details). It is seen to be good intermediate asymptotics for Leidenfrost droplets when on one hand, the droplet is still too large for a possible take-off [13], now Ref. [21] in this erratum, but on the other hand, already small enough to become nearly spherical.

Finally, we note that now that the numerical mistake has been corrected we generally find a really weak sensitivity of the results to the choice of the patching point $r=R_{p}$, e.g., $<0.1 \%$ when $R_{p}$ is varied between the values such that $h^{\prime}\left(R_{p}\right)=\tan (0.4 \pi)$ and $h^{\prime}\left(R_{p}\right)=\tan (0.45 \pi)$. This underscores the accuracy of our approach. However, such sensitivity is found to rapidly degrade in a vicinity of the leftmost limit of Figs. 5(c) and 5(d) for smaller droplets where it can reach several percent. For even smaller sizes as the Leidenfrost droplet is becoming well spherical, the result represented by the solid line, and Eq. (A1) is deemed to yield a better take than the present patching-point-based approach.

${ }^{1}$ This mistake did not propagate into post-2015 works of the present authors.

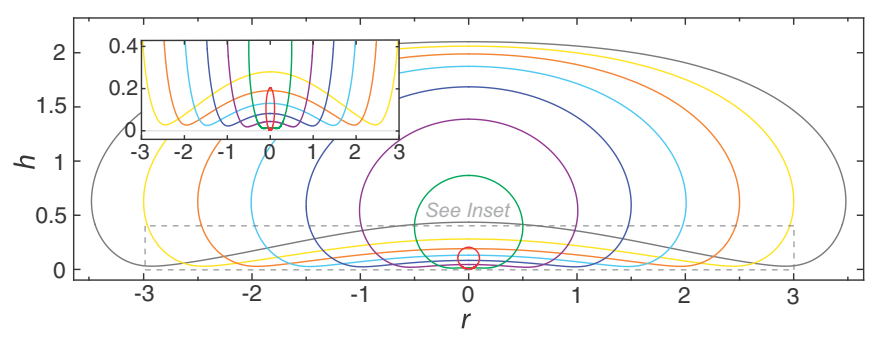

FIG. 2. Numerically determined shapes of Leidenfrost drops for $R_{\max }=0.1,0.5,1,1.5,2,2.5,3$, and 3.5 and $\mathcal{E}=1.21 \times 10^{-6}\left(T_{w}^{*}=\right.$ $370^{\circ} \mathrm{C}$ for water). The lengths are scaled by the capillary length $\ell_{c}^{*}=\left(\gamma^{*} / \rho_{\ell}^{*} g^{*}\right)^{1 / 2}$ (i.e., $2.5 \mathrm{~mm}$ for water). 


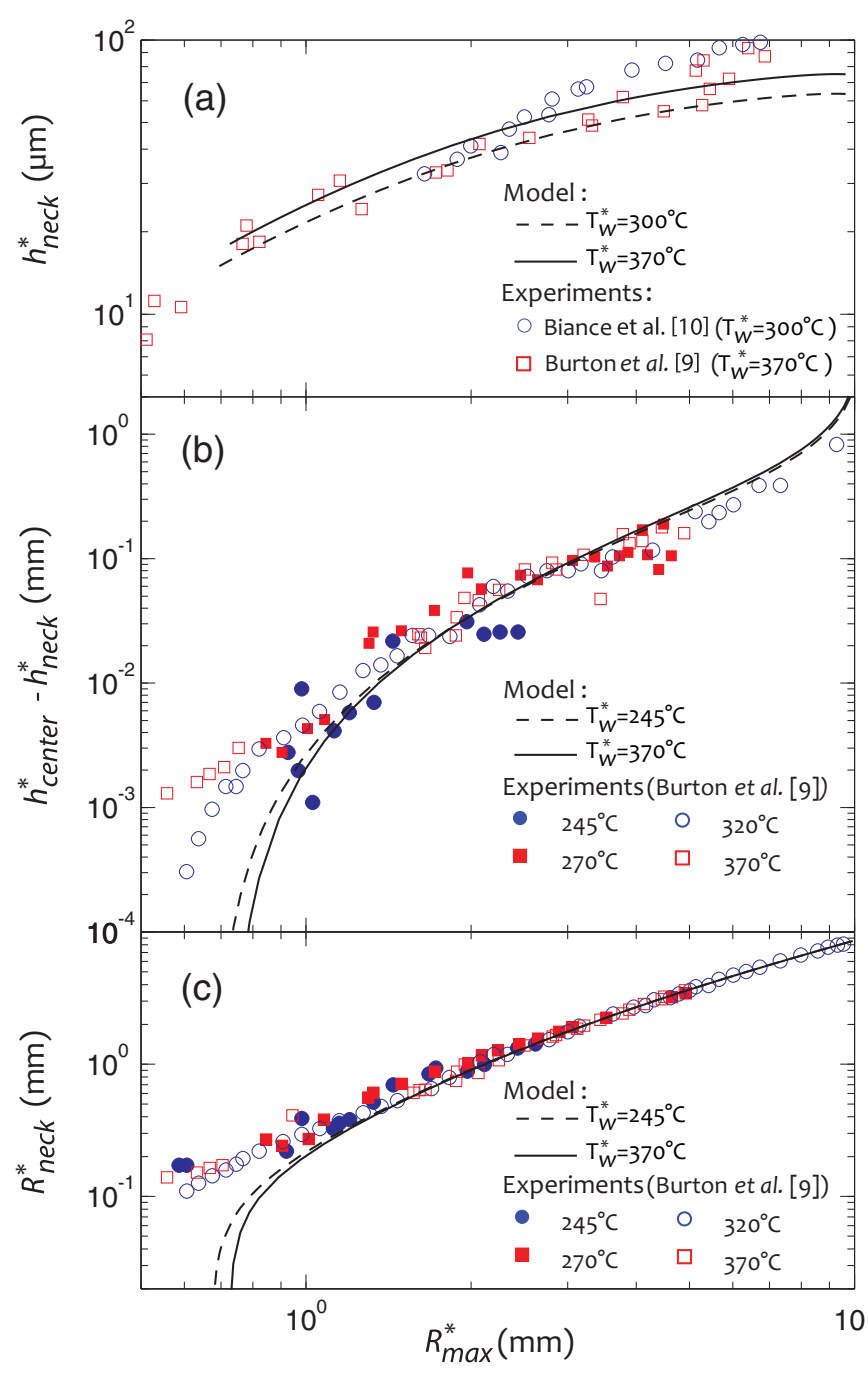

FIG. 3. Numerical calculations compared to experimental measurements (Burton et al. Ref. [9] of the original paper, now Ref. [18] in this erratum and Biance et al. Ref. [10] of the original paper, now Ref. [19] in this erratum) of the characteristics of the vapor layer under a Leidenfrost water drop. (a) The film thickness at the neck, (b) the depth of the vapor pocket, and (c) the neck radius are plotted as a function of the drop size $R_{\max }^{*}$ (the capillary length is $\ell_{c}^{*}=2.5 \mathrm{~mm}$ ).

(a)

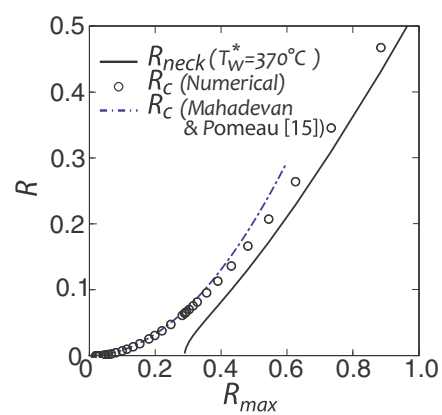

(b)

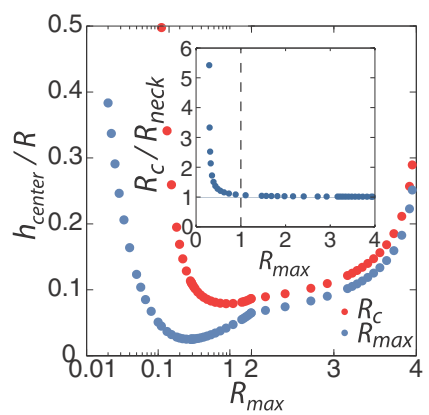

FIG. 4. (a) Radius at the neck $R_{\text {neck }}$ and static "contact" radius $R_{c}$ versus drop size $R_{\max }$ for $\mathcal{E}=1.21 \times 10^{-6}\left(T_{w}^{*}=370^{\circ} \mathrm{C}\right.$ for water). Note that Ref. [15] of the original paper is now Ref. [20] in this erratum. (b) Smallness parameter $h_{\text {center }} / R$ underlying the lubrication approximation versus $R_{\max }$ (it is $h_{\text {center }} / R_{c}$ for larger $R_{\max }$, but rather $h_{\text {center }} / R_{\max }$ for smaller $R_{\max }$, when the notion of $R_{c}$ ceases to be anyhow meaningful for the Leidenfrost droplet). The left part of the latter figure is plotted on a logarithmic scale for better visibility. The inset compares $R_{\text {neck }}$ and $R_{c}$ for a larger range of sizes than in (a). 

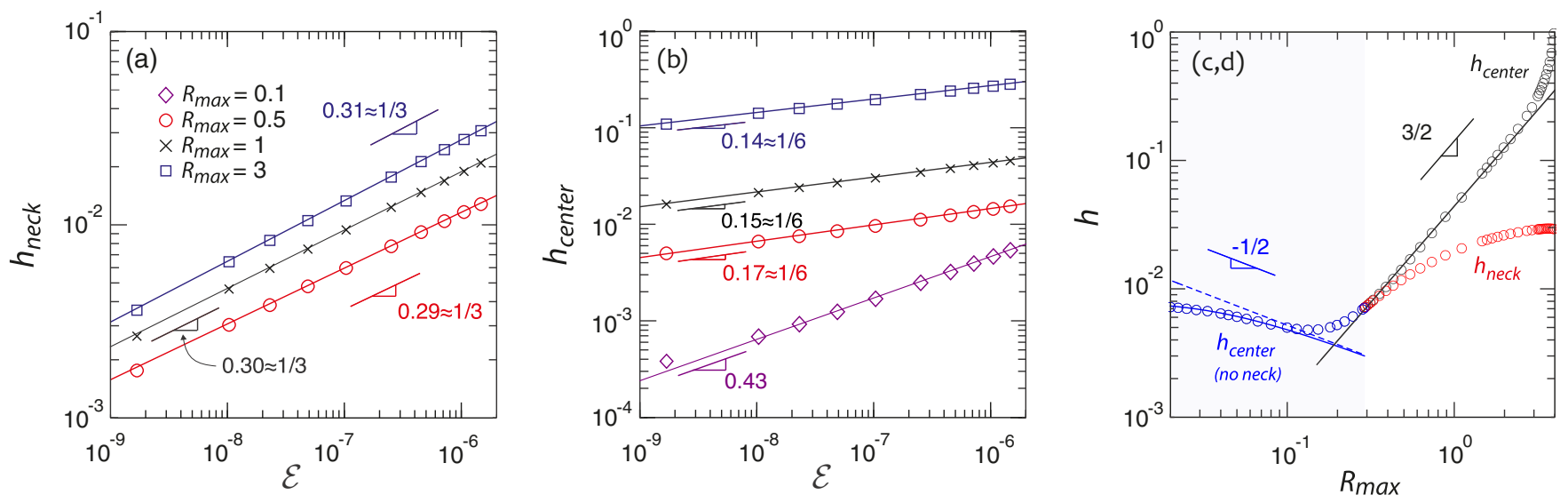

FIG. 5. Dimensionless vapor film thicknesses (a) at the neck and (b) at the center as a function of the evaporation number for various drop sizes. (c) and (d) (Combined in the present erratum.) The same thicknesses versus the drop size for an evaporation number $\mathcal{E}=1.21 \times 10^{-6}$.

\section{APPENDIX: INTERMEDIATE ASYMPTOTICS FOR SMALL LEIDENFROST DROPLETS}

The solid curve in Figs. 5(c) and 5(d) is obtained as follows. Recall in our original paper that $P_{v}$ is the dimensionless (scale $\rho_{l}^{*} g l_{c}^{*}=\gamma^{*} / l_{c}^{*}$ ) excess pressure in the vapor layer underneath the droplet. This pressure maintains the Leidenfrost droplet in levitation, counterbalancing its weight. The corresponding dimensionless force balance can be written as $\frac{4}{3} \pi R_{\max }^{3}=$ $2 \pi \int_{0}^{R_{\max }} r P_{v} d r$. Integrating by parts and implying $P_{v}=0$ at $r=R_{\max }$ (i.e., no excess over the ambient pressure), the force balance can be rendered as $\frac{4}{3} \pi R_{\max }^{3}=-4 \pi \int_{0}^{R_{\max }} r^{2} \partial_{r} P_{v} d r$. Equation (3) (dimensionless) of the original paper can be rewritten as $\partial_{r}\left(r h^{3} \partial_{r} P_{v}\right)=-12 r \mathcal{E} / h$. It can be integrated assuming a spherical shape $h=h_{\text {center }}+R_{\max }-\left(R_{\max }^{2}-r^{2}\right)^{1 / 2}$ to yield $\partial_{r} P_{v}$, which can, subsequently, be used in the force balance integral. Performing the two consecutive integrations analytically with the help of Wolfram Mathematica software, one can finally obtain the equation,

$$
\frac{R_{\max }}{h_{\text {center }}+R_{\max }}\left(\frac{R_{\max }^{2}}{h_{\text {center }}^{2}}-3 \frac{R_{\max }}{h_{\text {center }}}-6\right)+6 \ln \frac{h_{\text {center }}+R_{\max }}{h_{\text {center }}}=\frac{4 R_{\max }^{3}}{9 \mathcal{E}},
$$

which can numerically be solved to obtain $h_{\text {center }}$ for any given $R_{\max }$ and $\mathcal{E}$ as for the solid curve in Figs. 5(c) and 5(d). The leading-order approximation thereof yields

$$
h_{\text {center }}=\frac{3 \mathcal{E}^{1 / 2}}{2 R_{\max }^{1 / 2}}
$$

(c)

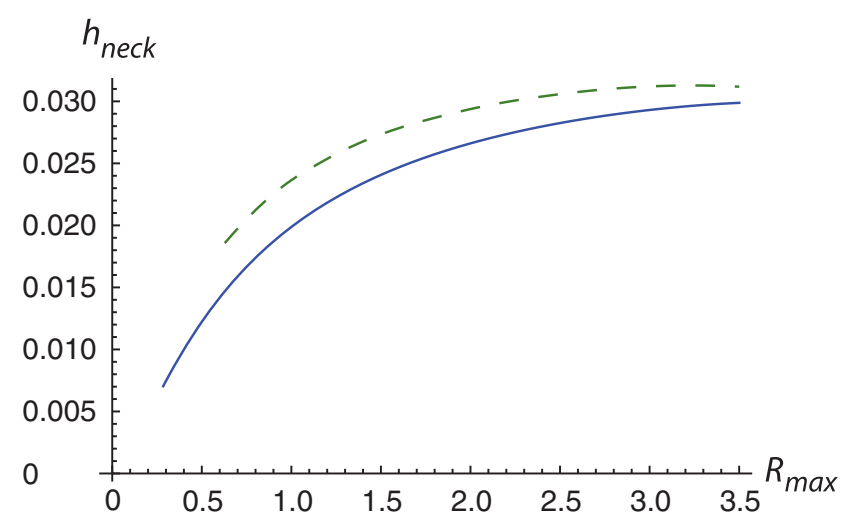

(d)

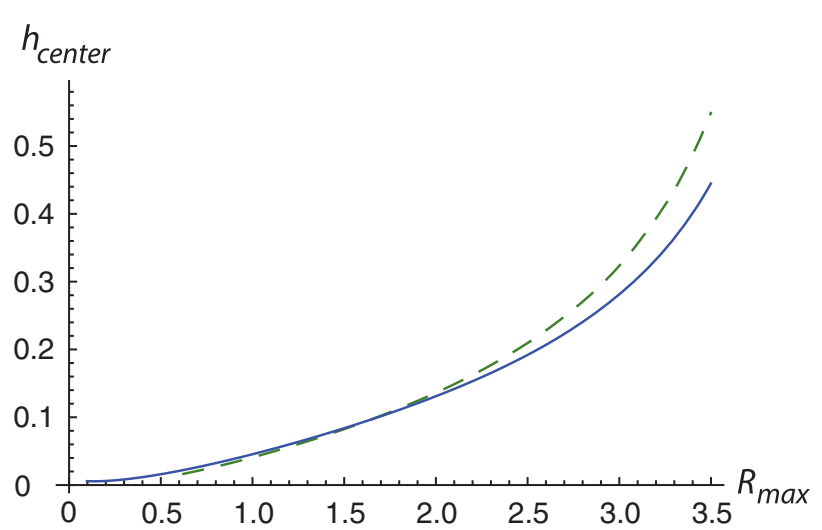

FIG. 7. Various quantities as functions of the maximum horizontal radius of the drop for $\mathcal{E}=1.21 \times 10^{-6}$ (water at $T_{w}^{*}=370^{\circ} \mathrm{C}$ ) when relevant. (c) $h_{\text {neck }}$, results of the asymptotic analysis as in Sec. V of the original paper (dashed, green line) and of the computation as in Sec. III of the original paper (solid, blue line). (d) $h_{\text {center }}$ with the line formats meaning the same as above. Modified with respect to our paper are the solid lines (matched asymptotic expansion results, dashed lines, unchanged). Now deemed impertinent, the dot-dashed (red) line originally present in our paper is not reproduced. 
corresponding to the dashed line in Figs. 5(c) and 5(d). Even if an ultimate asymptotic behavior of a spherical Leidenfrost particle in the limit $h_{\text {center }} \ll R_{\max }$, we see that Eq. (A2), unlike Eq. (A1), does not procure much overlap with the behavior of the Leidenfrost droplet. Indeed, where Eq. (A2) works well for a spherical particle, the Leidenfrost droplet is not sufficiently spherical. Inversely, where the Leidenfrost droplet is sufficiently spherical, the inequality $h_{\text {center }} \ll R_{\max }$ is not strong enough, and Eq. (A2) is better to be replaced with a more precise Eq. (A1).

[17] I. Chakraborty, M. V. Chubynsky, and J. E. Sprittles, Computational modeling of quasistatic Leidenfrost drops, Bulletin of the American Physical Society, 73rd Annual Meeting of the APS Division of Fluid Dynamics, Chicago, 2020 (APS, Ridge, NY, 2020).

[18] J. C. Burton, A. L. Sharpe, R. C. A. van der Veen, A. Franco, and S. R. Nagel, Phys. Rev. Lett. 109, 074301 (2012).
[19] A.-L. Biance, C. Clanet, and D. Quéré, Phys. Fluids 15, 1632 (2003).

[20] L. Mahadevan and Y. Pomeau, Phys. Fluids 11, 2449 (1999).

[21] F. Celestini, T. Frisch, and Y. Pomeau, Phys. Rev. Lett. 109, 034501 (2012).

Correction: An error in the logarithmic term on the left-hand side of Eq. (A1) has been fixed. 